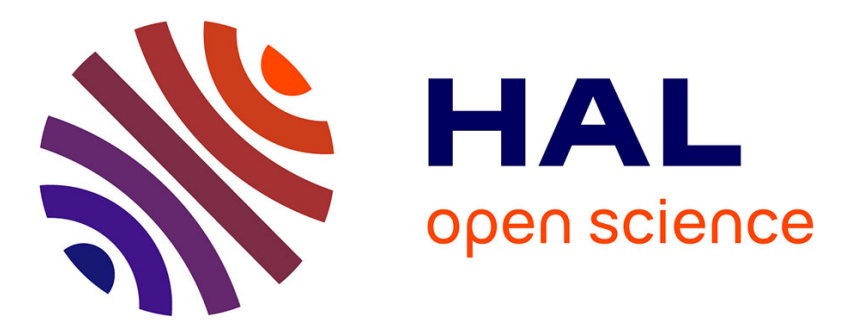

\title{
Characteristics of the DEGAS-FATIMA Hybrid setup for the DESPEC program at NUSTAR
}

\author{
G.S Li, Radomira Lozeva, I. Kojouharov, Jürgen Gerl, Magda Gorska
}

\section{To cite this version:}

G.S Li, Radomira Lozeva, I. Kojouharov, Jürgen Gerl, Magda Gorska. Characteristics of the DEGASFATIMA Hybrid setup for the DESPEC program at NUSTAR. Nuclear Instruments and Methods in Physics Research Section A: Accelerators, Spectrometers, Detectors and Associated Equipment, 2021, 10.1016/j.nima.2020.164806 . hal-03004343

\section{HAL Id: hal-03004343 https://hal.science/hal-03004343}

Submitted on 24 Nov 2020

HAL is a multi-disciplinary open access archive for the deposit and dissemination of scientific research documents, whether they are published or not. The documents may come from teaching and research institutions in France or abroad, or from public or private research centers.
L'archive ouverte pluridisciplinaire HAL, est destinée au dépôt et à la diffusion de documents scientifiques de niveau recherche, publiés ou non, émanant des établissements d'enseignement et de recherche français ou étrangers, des laboratoires publics ou privés. 


\title{
Characteristics of the DEGAS-FATIMA Hybrid setup for the DESPEC program at NUSTAR
}

\author{
G.S. Li ${ }^{\mathrm{a}}$, R. Lozeva ${ }^{\mathrm{b}, *}$, I. Kojouharov ${ }^{\mathrm{c}}$, J. Gerl $^{\mathrm{c}}$, M. Górska $^{\mathrm{c}}$ \\ ${ }^{a}$ Key Laboratory of High Precision Nuclear Spectroscopy and Center for Nuclear Matter \\ Science, Institute of Modern Physics, Chinese Academy of Sciences, Lanzhou, 73000, China \\ ${ }^{b}$ Université Paris-Saclay, CNRS/IN2P3, IJCLab, 91405 Orsay, France \\ ${ }^{c}$ GSI Helmholtzzentrum für Schwerionenforschung GmbH, 64291 Darmstadt, Germany
}

\begin{abstract}
We report on a study of the performance of the DEGAS-FATIMA Hybrid setup through GEANT4 simulations using accurate geometries of all detector components. We give details on the most compact detector configuration considered for this setup when maximising efficiencies of both DEGAS (HpGe) and FATIMA $\left(\mathrm{LaBr}_{3}(\mathrm{Ce})\right)$ systems with respect to a heavy-ion implantation and $\beta$-decay station. The possible configurations of the implantation system are discussed in terms of thickness variation and individual positioning of detector components. We provide guidelines for the Hybrid setup for the first NUSTAR/DESPEC experiments.
\end{abstract}

Keywords: DEGAS, FATIMA, photo-peak efficiency, add-back factor, coincidence spectra

\section{Introduction}

The DESPEC (DEcay SPECtroscopy) experiments [1] have recently started as a part of the NUSTAR (NUclear STructure, Astrophysics and Reactions) physics program [2, addressing the investigation of exotic nuclei produced in 5 heavy-ion induced fragmentation and fission reactions.

\footnotetext{
${ }^{*}$ Corresponding author

Email address: radomira.lozeva@ijclab.in2p3.fr (R. Lozeva)
} 
Coupled to the FRS (FRagment Separator) within FAIR Phase-0 and SuperFRS with FAIR Phase-1 [3], various detection setups are considered and constructed, including high-resolution $\gamma$-ray spectroscopy (DEGAS) 4, highefficiency decay spectroscopy (DTAS) [5], $\beta$-delayed neutron detection (BELEN) 10 [6, nuclear moments (gSPEC) [7, as well fast-timing measurements (FATIMA) 8. In this article we present a new idea of combining the DESPEC detector to a Hybrid system combining FATIMA and DEGAS in a new geometry, aiming at a precise $\gamma$-ray and fast-timing spectroscopy of isomers, surviving the flight path through the in-flight separator FRS, or at states populated after the $\beta$-decay of the implanted nuclei. Such system, thus, provides access to simultaneous $\gamma$ - $\gamma$-timing and $\beta$ - $\gamma$-timing spectroscopy of very exotic nuclei from light, intermediate to heavy species, unique to the FAIR facility. Being the subject of several DESPEC physics motivations, the interest is spanned from closed nuclear shells, excitation regimes, deformation and shapes to Gamow-Teller transitions and the astrophysical r-process in the description of the universe.

To prove the adequacy of the above setup, we present the simulation results for the DEGAS + FATIMA arrays in terms of configurations and efficiency. It includes the positioning of the $\gamma$-detection system, surrounding an ion implantation and $\beta$-detection station. This new Hybrid system allows simultaneously 25 a standard $\gamma$-ray spectroscopy using the HpGe detectors of DEGAS and a fasttiming $\gamma$-ray spectroscopy using the $\operatorname{LaBr}_{3}(\mathrm{Ce})$ scintillators of FATIMA. The spectroscopic information is tagged by the implantation of a heavy ion e.g. after an isomeric decay, as well as heavy-ion followed by a $\beta$ disintegration e.g. traced with the Double-Sided Silicon Strip Detectors (DSSSD) of AIDA [9]. A good timing reference is assured by two beta-plastic (bPlast) detectors surrounding the Si decay station. Several possibilities for placements of the Ge clusters and the fast scintillators, including their exact position in the corresponding array of rings with respect to the entire implantation setup, are all taken into account. Detector encapsulations and housings are considered as precise as in

35 the technical drawings from where we have exported the detector components and geometry. In addition, the positioning, distances and thickness of the im- 
plantation detectors are taken into account in the simulations. As described in the next sections, the possible variation in the beam through a Gaussian distribution of the implanted species is also considered.

\section{2. Geometrical model and source considerations}

An overview of the simulated Hybrid configuration is shown in Fig. 1, with an intersection, indicating the extreme positions (minimum and maximum) of the snout assembly, as well as two focal (focus) positions for FATIMA and for DEGAS. The DEGAS array on the right hand-side consists of 12 triple HPGe 45 clusters, arranged in two rings at $29^{\circ}$ and $72^{\circ}$ at forward angles with respect to the beam axis. The distances between front face of the detector and DEGAS focal point are $160 \mathrm{~mm}$ for both rings. The FATIMA array on the left hand-side (Fig. 1 $1(\mathrm{a}, \mathrm{b})$ ) contains $36 \mathrm{LaBr}_{3}(\mathrm{Ce})$ detectors arranged in three rings, at $102^{\circ}$, $124^{\circ}$, and $140^{\circ}$ backward angles. The distances between the three rings (front face) and the focal point of the FATIMA first ring are $107 \mathrm{~mm}, 134 \mathrm{~mm}$, and $182 \mathrm{~mm}$, respectively. The FATIMA focal position, thus, corresponds to the first detector ring. The two-types of detector arrays DEGAS and FATIMA surround a snout, containing a set of detectors in the most compact geometry, intended to be used for implantation and $\beta$-decay tagging. These are composed of AIDA

${ }_{55}$ Si DSSSD in a stack, sandwiched by two fast bPlast detectors on both sides. In the simulation, the Hybrid assembly was fixed while moving the snout (see Fig. 1 (b)), together with the source, always located at the middle Si of AIDA. Various positions along the beam line were studied to simulate the performance such as $\gamma$-ray absorption and detector efficiency $(\epsilon)$.

In order to be as realistic as possible, thus closest to the experiment, we have considered most of the geometrical components of the setup. The simulated components are depicted in Fig. 2. The DEGAS cluster head includes three Ge capsules inside the end-cap and the head lid. The capsules, with $0,8 \mathrm{~mm}$ thick Aluminum (Al) housing, are fixed on a cold frame, connected to the cold finger.

${ }_{65}$ The thickness of the $\mathrm{Al}$ end-cap of each triple cluster is $1,5 \mathrm{~mm}$. The Ge crystal 

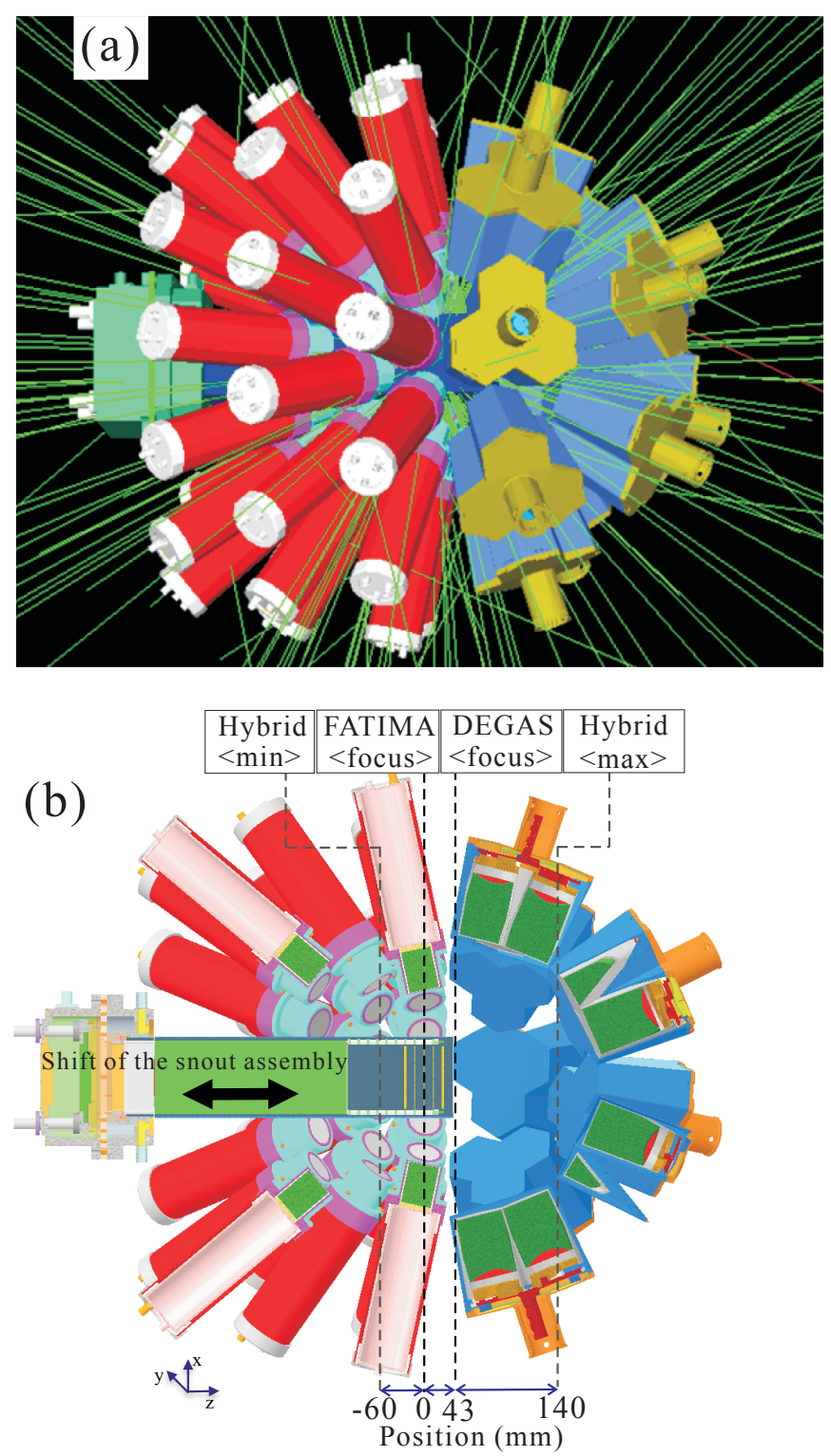

Figure 1: (a) View of the simulated Hybrid configuration. The green lines correspond to $\gamma$ rays. (b) Intersection of the setup with the possible positioning of the AIDA+bPlast station with respect to the DEGAS+FATIMA rings. In the simulation, the snout assembly was moved along the beam axis with respect to FATIMA and DEGAS in order to optimize the efficiency depending on the particular demands of experiments. 


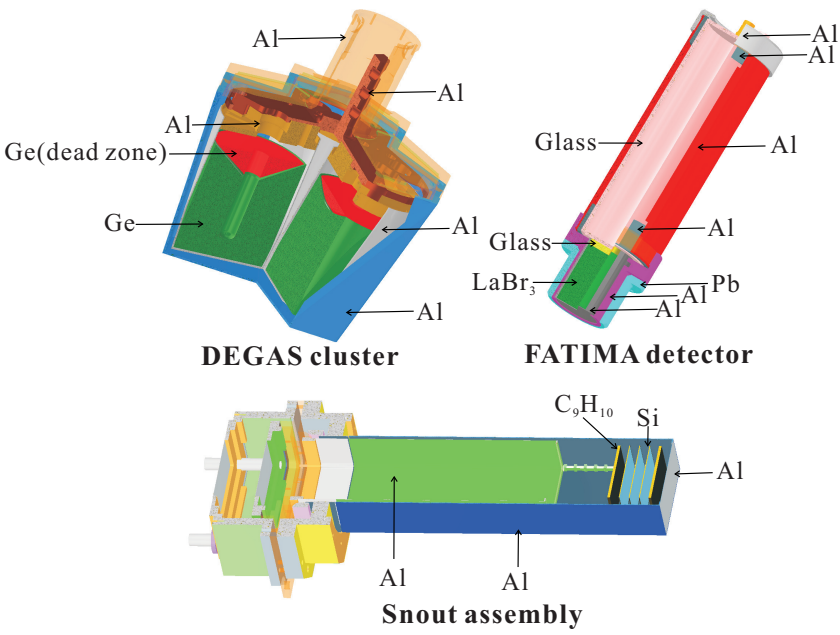

Figure 2: Components of a DEGAS cluster, a FATIMA $\mathrm{LaBr}_{3}(\mathrm{Ce})$ detector, and the snout assembly included in the simulation. See text for details.

has a diameter of $70 \mathrm{~mm}$ at the rear end and a length $78 \mathrm{~mm}$. To get a better comparison to the experimental data, a 10\% insensitive zone of conical shape at the rear part of the Ge crystal is assumed in the simulation [4. This assumption is based on the experience that the electrical field at the rear part is often found to be distorted when the biased Ge crystal is scanned. The phenomenon was explained by space charges on the open back surface of the detector [10]. The
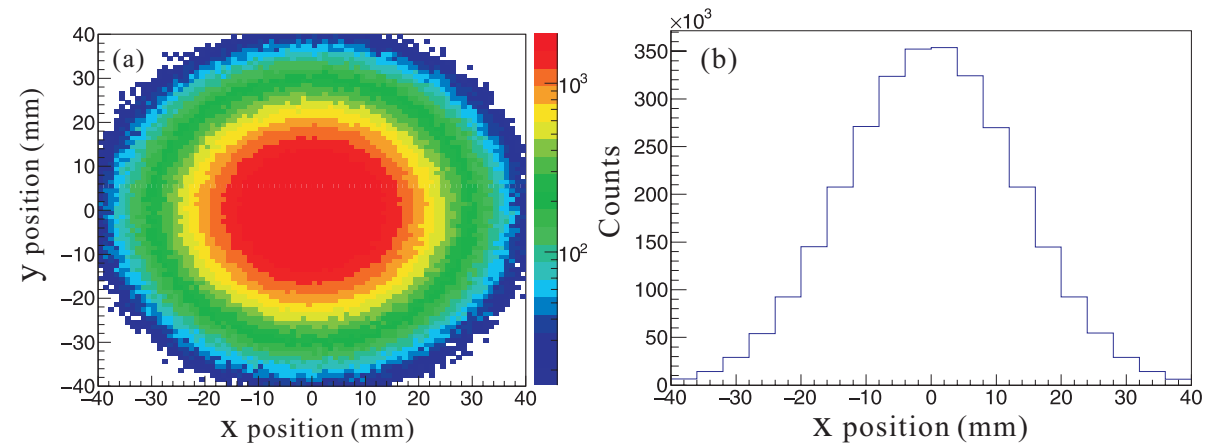

Figure 3: (a) Beam distribution ( $\mathrm{x}, \mathrm{y}$ ) over the Si AIDA detector as used in the Geant4 simulations. (b) Longitudinal $(x)$ projection from the Geant4 output. 
FATIMA $\mathrm{LaBr}_{3}$ crystal is cylindrical 2 inches in height, 1,5 inches in diameter, with $0,5 \mathrm{~mm}$ thick $\mathrm{Al}$ housing. The photomultiplier tube, optically coupled to the crystal is considered as a glass cylinder that is hollow inside. These active parts are packed inside an $\mathrm{Al}$ housing. A $5 \mathrm{~mm}$ thick lead cylinder is attached to the front cap as a passive shield. Inside the snout, the short version of AIDA [9] is considered. The basic unit of AIDA is a stack of three $80 \times 80 \mathrm{~mm}^{2}$ DSSSD, with $10 \mathrm{~mm}$ distance between them. Two Si detector thicknesses are selected in this study, namely of $0,5 \mathrm{~mm}$ and $1 \mathrm{~mm}$. Generally, up to ten Si detectors can be placed in the same snout depending on the experimental needs. The bPlast detectors are considered as thick as $3 \mathrm{~mm}$ with an area of $80 \times 80 \mathrm{~mm}^{2}$. The effect of two possible distances of the bPlast detectors with respect to the closest AIDA Si, such as $10 \mathrm{~mm}$ and $15 \mathrm{~mm}$, is studied. Mechanically, AIDA and bPlast are installed onto a support frame with four rods, that is also considered.

85 The housing of the snout is made of $\mathrm{Al}$ with a thickness of $0,3 \mathrm{~mm}$ in front and $1 \mathrm{~mm}$ in each of the side-directions.

To simulate a realistic situation, the $\gamma$ source is Gaussian distributed onto the central Silicon detector of AIDA such that in both $(x, y)$ directions at the FRS focal plane the beam spread covers the Si within 3 standard deviations of the source central position. Fig. 3 shows the used beam distribution over the Silicon detector centered in $(x, y)$ and its longitudinal $(x)$ projection. In the beam $(z)$ direction, the source position is centered at the middle Si detector (representing a beam implantation and respective radiation out of this detector). Mono-energetic $\gamma$ rays of twelve energies ranging from $30 \mathrm{keV}$ to $1408 \mathrm{keV}$ are used, and their detection further analysed.

\section{Implementation of the geometries and simulation}

\subsection{Add-back effect and efficiency response for different multiplicities}

In detecting the $\gamma$ rays, higher efficiency may be achieved by adding back the energies of Compton scattered events in different detectors. Typically, this 100 is important for the DEGAS clusters, with three HpGe crystals adjacent to 


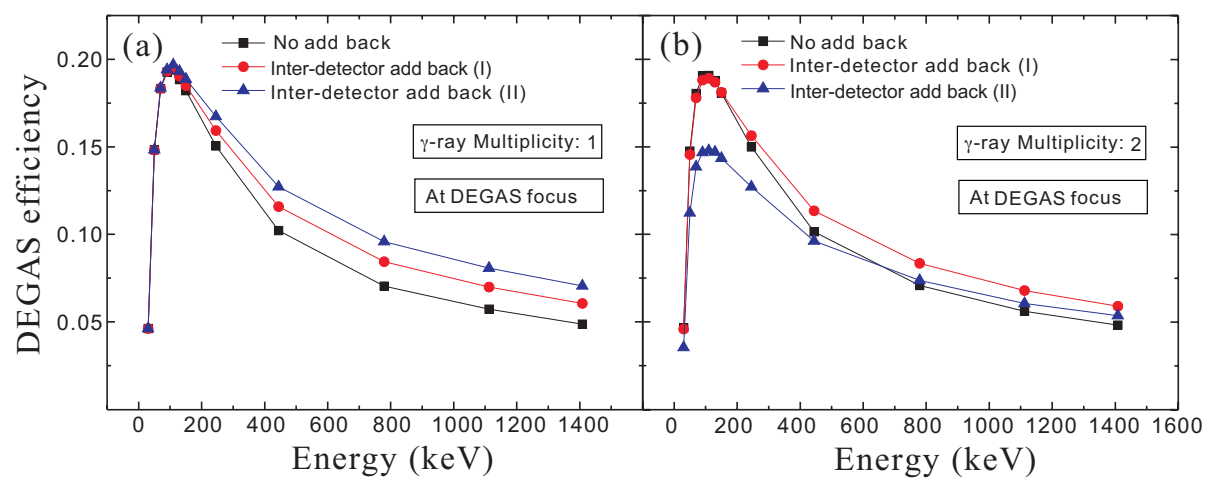

Figure 4: Efficiency curves for the DEGAS Hybrid array without add back, inter-detector add back (I), and inter-detector add back (II) for $\gamma$-ray multiplicity of $M=1$ (a) and $M=2$ (b). In the simulations, the middle Si detector of AIDA is located at the DEGAS focal point.

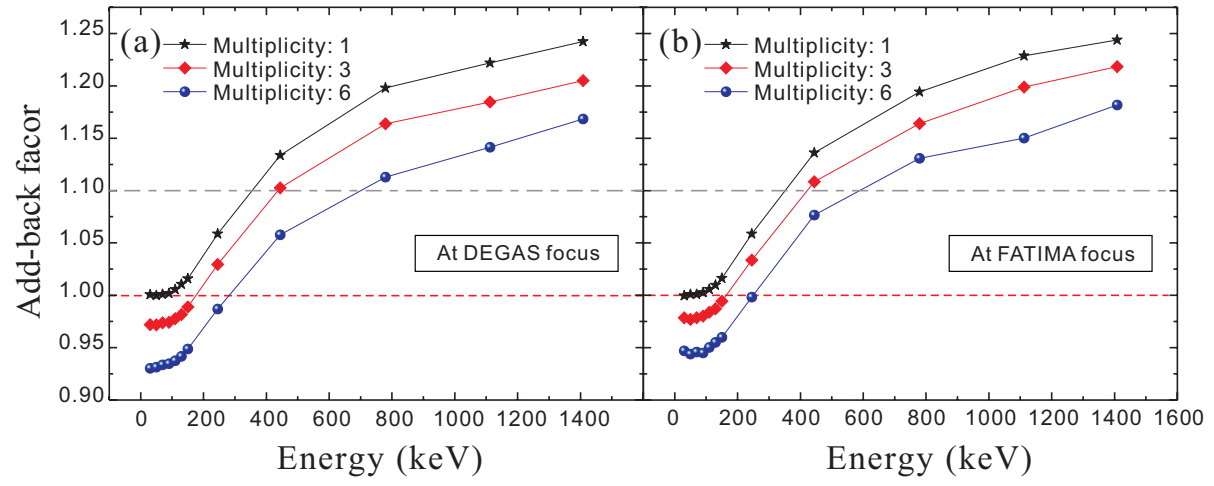

Figure 5: The inter-detector add-back (I) factor of DEGAS for different $\gamma$-ray multiplicities at DEGAS focus (a) and FATIMA focus (b). See text for details.

each other, where the probability that the full energy event is shared by two or even three crystals, is rather large. To study the add-back effect, a simulation is performed by positioning the Hybrid setup in such a way that the middle Si of AIDA is located at the DEGAS focal point (see Fig. 11). In this study, a stack of three $1 \mathrm{~mm}$ thick Si DSSSD detectors is chosen, and the distance between the DSSSD and the bPlast detector (front ones and back ones) is set to $10 \mathrm{~mm}$. As a starting point, it is assumed that the multiplicity of the $\gamma$ rays is one $(\mathrm{M}=1)$, corresponding to only one $\gamma$ ray emitted for each simulated event. 


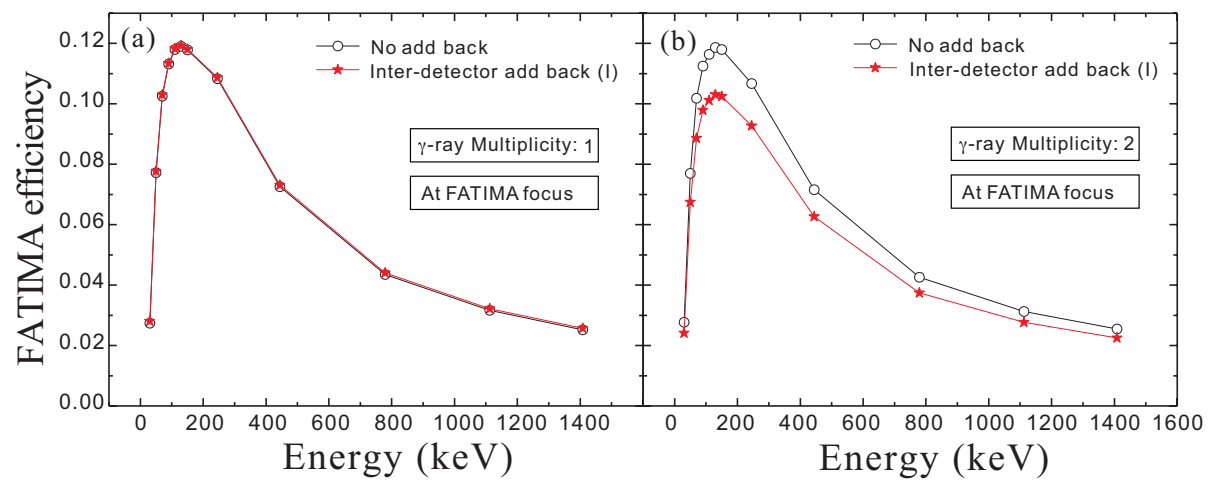

Figure 6: Efficiency curves for the FATIMA array without add back, and inter-detector add back (II) for the $\gamma$-ray multiplicity of $M=1$ (a) and $M=2$ (b). In the simulations, the middle Si detector of AIDA is located at the FATIMA focal point.

Table 1: Simulated DEGAS efficiencies for $M=1,3,6$ in the inter-detector add-back (I) mode at the DEGAS focal position. See text for details.

\begin{tabular}{cccccc}
\hline \hline $\mathrm{E}_{\gamma}(\mathrm{keV})$ & $\epsilon_{M=1}(\%)$ & $\epsilon_{M=3}(\%)$ & $\epsilon_{M=6}(\%)$ & $\left(\epsilon_{M 1}-\epsilon_{M 3}\right) / \epsilon_{M 1}$ & $\left(\epsilon_{M 1^{-}} \epsilon_{M 6}\right) / \epsilon_{M 1}$ \\
\hline 30 & 4,621 & 4,463 & 4,167 & 0,034 & 0,098 \\
50 & 14,818 & 14,226 & 13,322 & 0,040 & 0,101 \\
70 & 18,340 & 17,502 & 16,370 & 0,046 & 0,107 \\
90 & 19,312 & 18,353 & 17,300 & 0,050 & 0,104 \\
110 & 19,505 & 18,577 & 17,444 & 0,048 & 0,106 \\
130 & 19,039 & 18,244 & 17,138 & 0,042 & 0,100 \\
150 & 18,496 & 17,770 & 16,647 & 0,039 & 0,100 \\
245 & 15,936 & 15,243 & 14,325 & 0,043 & 0,101 \\
444 & 11,583 & 11,115 & 10,472 & 0,040 & 0,096 \\
779 & 8,445 & 8,148 & 7,588 & 0,035 & 0,102 \\
1112 & 6,997 & 6,578 & 6,162 & 0,060 & 0,119 \\
1408 & 6,051 & 5,807 & 5,447 & 0,040 & 0,100 \\
\hline \hline
\end{tabular}

The extracted results, with and without add back, are presented in Fig. 4 (a).

One can see that for low energy $\gamma$ rays, there is almost no improvement in the 
Table 2: Simulated FATIMA efficiencies for $\mathrm{M}=1,3,6$ at FATIMA focus position. No inter-detector add back is applied to these efficiencies.

\begin{tabular}{cccccc}
\hline \hline $\mathrm{E}_{\gamma}(\mathrm{keV})$ & $\epsilon_{M=1}(\%)$ & $\epsilon_{M=3}(\%)$ & $\epsilon_{M=6}(\%)$ & $\left(\epsilon_{M 1}-\epsilon_{M 3}\right) / \epsilon_{M 1}$ & $\left(\epsilon_{M 1}-\epsilon_{M 6}\right) / \epsilon_{M 1}$ \\
\hline 30 & 2,753 & 2,750 & 2,728 & 0,001 & 0,009 \\
50 & 7,736 & 7,601 & 7,562 & 0,017 & 0,023 \\
70 & 10,220 & 10,121 & 9,981 & 0,010 & 0,023 \\
90 & 11,322 & 11,124 & 11,089 & 0,018 & 0,021 \\
110 & 11,635 & 11,646 & 11,466 & $-0,001$ & 0,015 \\
130 & 11,903 & 11,786 & 11,586 & 0,010 & 0,027 \\
150 & 11,834 & 11,741 & 11,592 & 0,008 & 0,020 \\
245 & 10,785 & 9,325 & 10,537 & 0,002 & 0,023 \\
444 & 7,195 & 7,162 & 7,108 & 0,005 & 0,012 \\
779 & 4,315 & 4,330 & 4,222 & $-0,004$ & 0,021 \\
1112 & 3,176 & 3,091 & 3,042 & 0,027 & 0,042 \\
1408 & 2,539 & 2,470 & 2,463 & 0,027 & 0,030 \\
\hline \hline
\end{tabular}

add-back mode, as most of the $\gamma$ rays are deposited only in one HpGe crystal. For the higher energy $\gamma$ rays, as the Compton scattering between the crystals is more likely, there is an evident increase of efficiency when the add back between the inner-cluster crystals, called here inter-detector add back (I), is employed. The efficiency could be further improved by applying the algorithm of add back between the inner-cluster crystals and the different clusters, called here interdetector add back (II) (see Fig. 4(a)). We employ an add-back factor, defined as the gain of photo-peak efficiency in add-back mode with respect to the summed photo-peak response of individual detector, to check the performance. For the $1408-\mathrm{keV} \gamma$ ray, the factor is of 1,24 with inter-detector add back (I) and could reach up to 1,45 in inter-detector add back (II).

This analysis is important, taking into account that often in the experiments, e.g. for isomers, high-multiplicity events may be registered. For DEGAS it is ex- 
tremely relevant in case of building up level schemes, when events with minimum $\mathrm{M}=2$ are needed for the construction of $\gamma-\gamma$ matrices. For intermediate-spin states, that can generally be populated [11], $\mathrm{M}=6$ may be considered as kind of an extreme case, despite the naturally possible higher multiplicities. Events after $\beta$-decay also require $\mathrm{M}>1$. Therefore, relevant reduction in efficiency has to be taken into account, and the add-back factor needs further investigation.

${ }_{130}$ For a relative comparison, we also simulate the Hybrid performance with $\mathrm{M} \geq$ 2 at the DEGAS focal point. In the $\mathrm{M}=2$ case, taking the determination of the efficiency at $1408 \mathrm{keV}$ as an example, we consider two $\gamma$ rays emitted in each event. One of these is the $1408-\mathrm{keV} \gamma$ ray and the other one is randomly chosen among the twelve mono-energetic $\gamma$ rays ranging from $30 \mathrm{keV}$ to 1408 $\mathrm{keV}$. Similarly, in the $\mathrm{M}=3$ case, three $\gamma$ rays are emitted in each event. For the same example, one is the $1408-\mathrm{keV} \gamma$ ray and the other two are randomly taken. The resulting efficiency curves in the simulation for $\mathrm{M}=2$ are shown in Fig. 4 (b). As the Compton scattering between the inter-cluster crystals is less likely, the add-back algorithm will cause "false" summing events when the $\gamma$-ray multiplicity is higher, and thus resemble an efficiency drop at low energy region (see e.g. the trend of blue triangles in Fig. 4 (b)). Simulation runs performed with a ${ }^{60} \mathrm{Co}$ source $(\mathrm{M}=2)$ at the DEGAS focus revealed that the DEGAS efficiency for the $1332.5-\mathrm{keV} \gamma$ ray is $4.97 \%$ in a single detector mode, with inter-detector add back (I) the efficiency increases to $6.06 \%$, whereas the number sits at $5.57 \%$ when the inter-detector add-back (II) algorithm is applied. Therefore, we only chose inter-detector add back (I) for the further analysis of the data from the DEGAS HpGe array. Additionally, Fig. 5 (a) shows the interdetector add-back (I) factors for different $\gamma$-ray multiplicities using respective efficiencies from single crystals without add back as references. For the highenergy $\gamma$ rays, scattering from one crystal to its neighbour is quite likely and the add-back factor could still be more than one, whereas for low energy $\gamma$ rays, scattering is less likely and the increased possibility of "false" $\gamma$-ray summing would actually reduce the add-back factor to less than one. A DEGAS addback factor study is performed also at the FATIMA focus, and the results are 
focus, with a slight increase for $\mathrm{M}=3$ and $\mathrm{M}=6$. The simulated DEGAS inter-detector add-back (I) efficiencies for $\mathrm{M}=1,3,6$ (at the DEGAS focal position) are shown in Table 1. Overall, the efficiency drop is about 4\% (relative percentage) when the multiplicity is increased from 1 to 3 , and about $10 \%$

presented in Fig. 5(b). A similar behaviour could be seen as at the DEGAS (relative percentage) drop is predicted when the multiplicity is increased to 6 . Accordingly, at the FATIMA focal position, DEGAS efficiency drops of about $3 \%$ and $8 \%$ are predicted, for multiplicities 1 to 3 and 1 to 6 , respectively. Note that $\mathrm{M}=6$ is the highest we consider here, however, in specific cases it may be higher. It has to be clear that for a $\gamma$ ray multiplicity of $\mathrm{M}=6$ in case of Compton scattering an even higher crystal-hit multiplicity may occur.

Similarly for the $\mathrm{LaBr}_{3}$ detectors, $\mathrm{M}=2$ events are important for the analysis of short lifetimes, thus the respective reduction in efficiency needs an investigation. Fig. 6 shows efficiency curves at $M=1$ (a) and $M=2$ (b) for cases without any add back and inter-detector add-back (II) mode which can be simulated as well. In the simulation, the middle Si of AIDA is set at the FATIMA focal point. As it can be seen at $M=1$, only a little gain in the efficiency is present when the add-back algorithm between different detectors is applied. This is expected since the distances between the detectors are much larger than those of the DEGAS clusters, and due to the Pb shielding the probability of Compton-shared events is low. As shown in Fig. 6(b), the add-back mode effectively reduces the efficiencies due to the "false" summing event. The efficiency at $1.3 \mathrm{MeV}\left({ }^{60} \mathrm{Co}\right)$ according to the simulation is $2.62 \%$ without add back, while the number drops to $2.36 \%$ when inter-detector add-back (II) mode is employed. Therefore, no add back is suggested in the analysis of data from FATIMA array when $M>1$.

Table 2 presents the simulated FATIMA efficiencies for $\mathrm{M}=1,3$, and 6 at FATIMA focal position in a single detector mode. For example, it is visible that, due to the low efficiency compared to the DEGAS array, the medium drop is only about $2.2 \%$ (relative percentage) even at $\mathrm{M}=6$. With the FATIMA efficiency study at the DEGAS focal position, about $2.4 \%$ (relative percentage) medium drop is predicted when assuming multiplicities from $\mathrm{M}=1$ to $\mathrm{M}=6$. 


\subsection{Coincidence spectra}

In order to provide a guideline to estimate coincidence probabilities, another realistic simulation is further performed. A total number of $1 \times 10^{6}$ $\mathrm{M}=3$ events are simulated, each event consisting of a 344-779-1299 $\mathrm{keV} \gamma$-ray sequence. These energies are chosen to cover the main detectable energy range of the FATIMA array. The detector resolution is considered as a result of redistributing the simulated data of each event with a random variable, biased on a Gaussian distribution with a standard deviation [8, 12]

$$
\sigma=\frac{E^{1 / 2}}{a}
$$

where $E$ refers to the energy of the $\gamma$ ray in $\mathrm{keV}$ and $a$ is a constant. The

fitted values from data on a DEGAS cluster and a FATIMA detector of $a=$

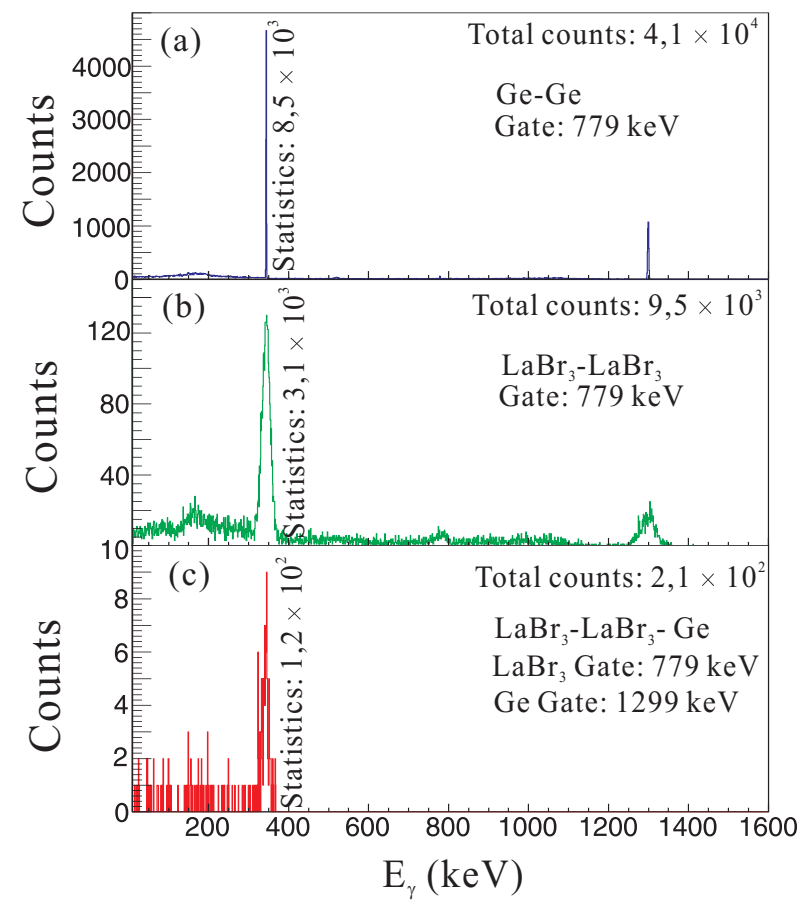

Figure 7: Simulated gated spectra for Ge-Ge (a), $\mathrm{LaBr}_{3}-\mathrm{LaBr}_{3}$ (b), as well as $\mathrm{LaBr}_{3}-\mathrm{LaBr}_{3}-$ Ge (c) coincident cases. In the simulation, $1 \times 10^{6} \mathrm{M}=3$ events are assumed, each event consists of the 344-779-1299 $\mathrm{keV} \gamma$-ray sequence, emitted at the DEGAS focal point. 
$36.5 \mathrm{keV}^{-1 / 2}$ for a HPGe detector and $2.5 \mathrm{keV}^{-1 / 2}$ for a LaBr3 detector, are the standard resolutions for these detectors and are used in the simulation. One can note, that some deviation may exist when using the equation (1) to describe the detector energy resolution in the experiment. However, the effect of the inaccuracy of this approximation is of lesser entity than that related to the level of statistical significance, regarding the efficiency we investigate here. The resulting Ge-Ge (gate: $779-\mathrm{keV}$ ), $\mathrm{LaBr}_{3}-\mathrm{LaBr}_{3}$ (gate: $779-\mathrm{keV}$ ), as well as $\mathrm{LaBr}_{3}-\mathrm{LaBr}_{3}-\mathrm{Ge}\left(\mathrm{LaBr}_{3}\right.$ gate: $779-\mathrm{keV}$; Ge gate: 1299-keV) coincidence spectra are shown in Fig. 7. In the Ge-Ge and $\mathrm{LaBr}_{3}-\mathrm{LaBr}_{3}$ coincidence spectra, requiring two-fold events, the statistics for the $344-\mathrm{keV}$ transition amounts to $8,5 \times 10^{3}$ and $3,1 \times 10^{3} \gamma$-ray counts, respectively. For the $\mathrm{LaBr}_{3}-\mathrm{LaBr}_{3}-\mathrm{Ge}$ coincidence spectrum, requiring three fold events, the statistics for the 344$\mathrm{keV} \gamma$ ray is about $1,2 \times 10^{2}$, which basically shows, as expected, an order of magnitude difference for any extra coincidence fold. It should be pointed out, that as no background is assumed in this simulation, the statistics results should be regarded as an upper limit. In addition, one should also take into account the DAQ dead time loses when comparing this simulation with real experimental data. Typically, in these multidetector systems the reduction is of the order of $20 \%$.

\subsection{The $\gamma$-ray absorption by detector housings, AIDA, and bPlast detector}

The $\gamma$-ray absorption is studied by fixing the Hybrid setup at the DEGAS and at the FATIMA focal positions (see Fig 1). In some experimental studies the efficiency drop at low energies is not an important factor but in others, it could be crucial. Especially in cases where the energies are below $100 \mathrm{keV}$ and 220 thus mostly converted, only a very small $\gamma$ part may be detected experimentally. One has to note, that due to the prompt flash, the low-energy region is often cut, thus the low-energy part is generally a compromise between the different factors and depends on the experimental needs. From a point of view of absorption of the low-energy $\gamma$ rays due to their passage trough materials and detector housings, the question can still be investigated. For example, the Si detectors, 


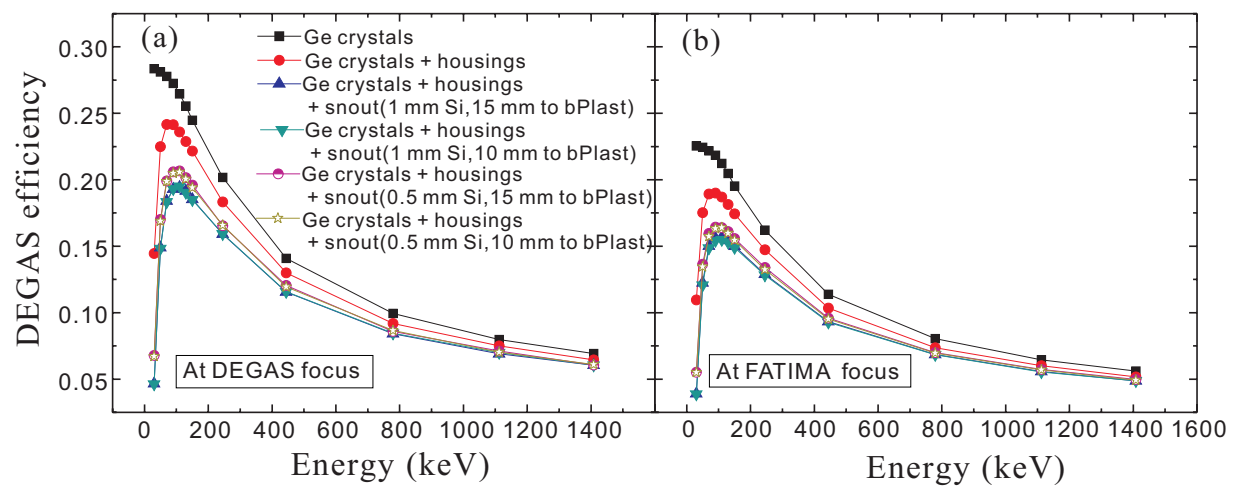

Figure 8: The simulated efficiency curves of the DEGAS setup in various cases at DEGAS (a) and at FATIMA (b) focuses.

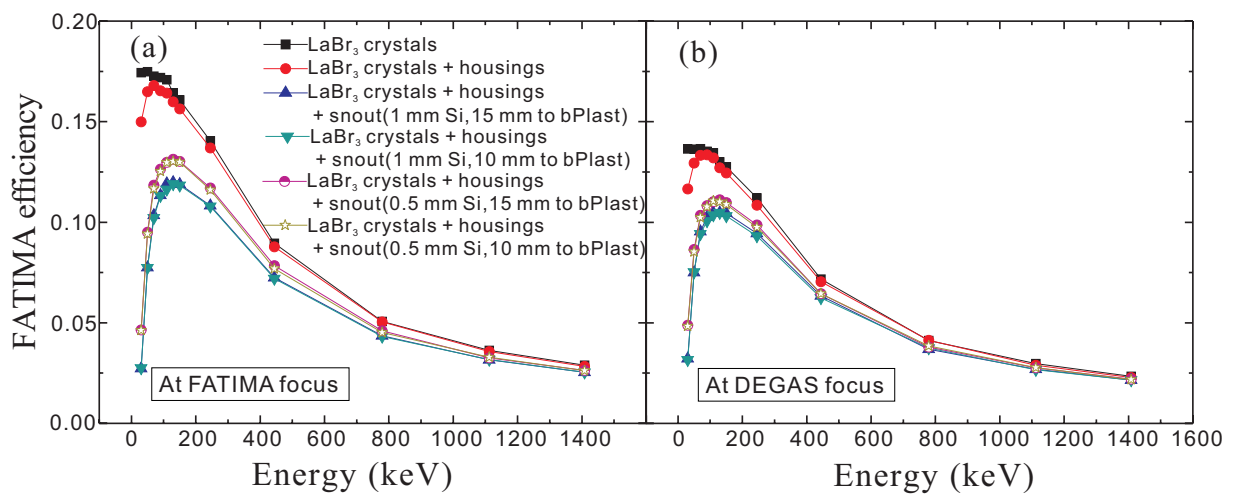

Figure 9: The simulated efficiency curves of the FATIMA setup in various cases at FATIMA (a) and at DEGAS (b) focuses.

used as an implanter, attenuate the low-energy $\gamma$ rays. Some minimum $\mathrm{Si}$ thickness is needed to generate sufficiently large signals from the $\beta$ particles in the DSSSD detector above the noise, to be able to discriminate it. The very thick Si detectors have lower efficiency than the thin ones, as shown in another Geant4 simulation for various heavy ion species [13]. On the other hand, independent of the individual Si detectors a certain total thickness (of the stack) is needed to assure stopping of all ions. Therefore, a case dependent compromise has to be found, including the detection range needed to be covered for the species of interest (the reaction $\mathrm{Q}$ value etc.). Here, we investigate the 


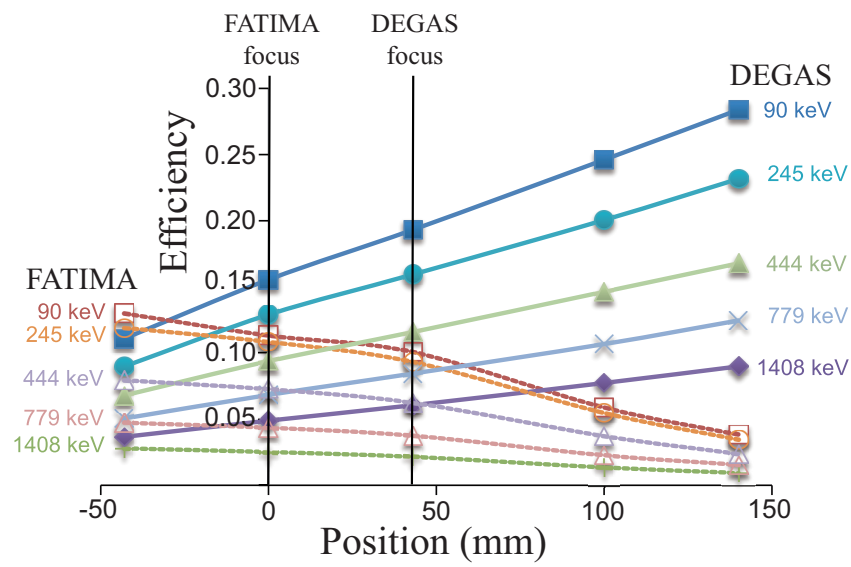

Figure 10: Simulated efficiency trends of DEGAS and FATIMA arrays with moving the snout assembly along the beam axis. See text for details.

effect for $0,5 \mathrm{~mm}$ and $1 \mathrm{~mm}$ thickness of the Si detectors, and the effect for $10 \mathrm{~mm}$ and $15 \mathrm{~mm}$ distance to the bPlast detectors, taking into account that the thickness of the detectors housings is fixed. The result can be seen in Fig. 8, showing the simulated DEGAS efficiency curves with different conditions, corrected with inter-detector add back (I) at DEGAS and FATIMA focuses. These estimates seem realistic in some of the $\beta$ decay experiments combined with fast-timing. The efficiency for $0,5 \mathrm{~mm}$, compared to the $1 \mathrm{~mm}$ Si thickness is increased, whereas a little difference in the efficiency is expected for the 10 $\mathrm{mm}$ and $15 \mathrm{~mm}$ distances between bPlast and the AIDA detectors. As expected, the housings of the Ge crystals cause the most drastic efficiency drop at the low energy region, whereas the drop is less significant in the higher-energy region. The snout assembly lowers even further the efficiency at the low-energy region. Note that the simulation predicts similar reduction effect in the efficiency of the FATIMA array (see Fig 9 ) caused by the snout assembly and, as in the DEGAS case, may be taken as a limit for a low-energy $\gamma$-ray detection.

\subsection{Efficiency scan along the beam axis}

Further study is performed when moving the Hybrid detector arrays along the beam axis, to obtain their possible efficiencies at different positions. In 
this simulation, the thickness of the Si detectors is taken to be $1 \mathrm{~mm}$, while the distance from Si to the bPlast detector (front ones or back ones) is fixed to $10 \mathrm{~mm}$. The resulted efficiency trends for DEGAS and FATIMA arrays are shown in Fig. 10. The DEGAS curves are corrected with inter-detector add back (I) whereas the curves for FATIMA are from single detectors. From this study, when both DEGAS and FATIMA arrays are moved along the central (=beam) trajectory, one can determine the optimal placement depending on the experimental needs. For experiments where FATIMA fast-timing spectroscopy is the most important factor, the position which gives its maximum efficiency is needed. According to Fig. 10, this is the FATIMA focus position, corresponding to the first $\mathrm{LaBr}_{3}$ detector ring. It is the first ring that contributes mostly to the total FATIMA efficiency in this case, despite the fact that it contains the same number of detectors of the other rings. For this position, also reasonable efficiency from DEGAS detectors can be expected. Although FATIMA has slightly higher efficiency if the detectors are moved backwards, more extreme positions are technically possible e.g. to $-60 \mathrm{~mm}$ (corresponding to second $\mathrm{LaBr}_{3}$ ring placement), and some modification of the snout length may be necessary. Also, in that case, the efficiency of DEGAS is dropped as well, thus e.g. crosscheck of $\gamma$ rays for identification will be less effective. For example, the results of the simulation at the symmetrical to the DEGAS focus position $(-43 \mathrm{~mm})$, are shown in Fig. 10. Alternatively, for cases when DEGAS high-resolution spectroscopy is the more important factor but some lifetime spectroscopy is also desired from FATIMA, the DEGAS focal position $(+43 \mathrm{~mm})$ should be considered.

One can envisage moving the snout completely inside the DEGAS array along the snout assembly. Technically, the most extreme position, which we also consider in the Geant4 simulation is $+140 \mathrm{~mm}$. Depending on the experimental 280 needs, it should be noted that here only 12 DEGAS detectors are taken (e.g. corresponding to $9.0 \%$ at $1408 \mathrm{keV}$ in inter-detector add-back (I) mode). If even higher Ge efficiency is needed, going to the full DEGAS configuration would be more advantageous. In such configuration, e.g. for the wide version of AIDA 
composed of three horizontally $(x)$ placed $\mathrm{Si}$ (covering $+/-120 \mathrm{~mm}$ ) combined

$18 \%$ at $1,3 \mathrm{MeV}$ is reported in Ref. 4.

\subsection{Beam distribution and detector coverage}

In the presented simulations, the fragment distributions over the implantation detectors are assumed to be Gaussian. In the case of the single Si DSSSD with $(x, y)$ of $(80 \times 80) \mathrm{mm}^{2}$, the beam covers the entire detector as shown in Fig. 3 together with its longitudinal $(x)$ projection. Often several species are transmitted to this final focal plane, and their distributions are displaced in $x$, and wide in $x$, as this is the dispersive direction. In such cases the beam may need to be collimated prior the implantation station. In order to cover several isotopes of interest or wide distributions (e.g. in monochromatic FRS mode), a wide AIDA version of $3 \mathrm{Si}$ DSSSD covering of $(x, y)$ of $(240 \times 80) \mathrm{mm}^{2}$ may also be used.

These detectors will fit into the Hybrid ball if some of the close-lying scintillators are retracted from the implantation snout. A modified version of the snout may also be needed. In order to technically fit at the FATIMA focal position or the DEGAS focal position, the displacement of several detectors would result in a slight reduction in the respective efficiencies. From the technical drawings, it can be estimated that at the FATIMA focal position, the displacement of the FATIMA detectors needed is between $30 \mathrm{~mm}$ and $45 \mathrm{~mm}$ for 16 out of the $36 \mathrm{LaBr}_{3}(\mathrm{Ce})$ detectors. For the DEGAS detectors no displacement is needed, neither at the FATIMA focal position nor at the DEGAS focal position. Therefore, the DEGAS efficiencies for these two cases may be considered unchanged. For the FATIMA efficiencies at the FATIMA focus, it is estimated that the relative reduction factor for the efficiencies reported in Fig. 9 (a) is $16(1) \%$, taking into account that most of the detector positions can actually be kept, and the current space for placing the snout is reasonably large. In Fig. 11 such an example is shown for the FATIMA $\mathrm{LaBr}_{3}(\mathrm{Ce})$ detectors with casemates, and without the snout. Further movement of wide AIDA station inside DEGAS 

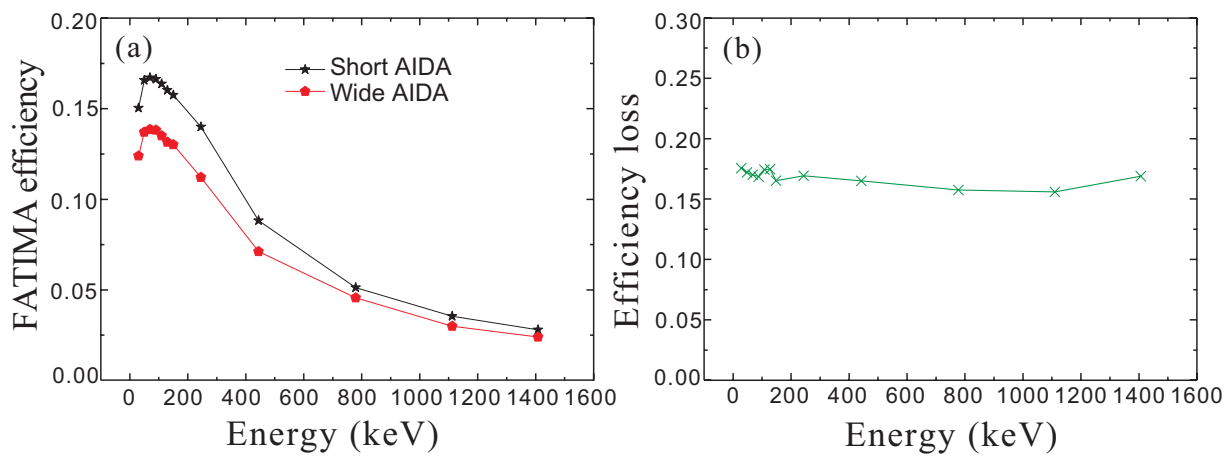

Figure 11: Simulated efficiency trends of FATIMA at the FATIMA focal position for short and wide AIDA (a) and the average reduction in efficiency (b). See text for details.

would require retraction of the DEGAS detector positions (e.g. 4 of the closest detectors) and can similarly be estimated according to the experimental needs.

\section{Summary}

The performance of a Hybrid detector array consisting of DEGAS, FATIMA, AIDA, as well as bPlast detectors is studied in the Geant4 simulation framework. We investigate various positions of the DEGAS and FATIMA detector arrays, the content of the implantation snout, the thickness of the implantation detectors and distances between them. We also study the effect of $\gamma$-ray multiplicities on the detector efficiencies, the beam distribution and $\gamma$-ray absorption in inactive detector materials and housing. This work can be used as a guideline for first DESPEC and FATIMA experiments at the FRS using the Hybrid configuration, and can in the future be compared to the experimental results from these upcoming works.

\section{ACKNOWLEDGEMENTS}

The work at Institute of Modern Physics, CAS, was supported by the Youth Innovation Promotion Association of Chinese Academy of Sciences (Grant No. 2019407), and the National Natural Science Foundation of China (Grants No. 
U1932138, U1732139). The work at Université Paris-Saclay was supported by the IN2P3-GSI collaboration agreements on DESPEC/gSPEC collaboration works on GSI/FAIR.

[1] RUBIO B. Decay spectroscopy (despec) at the new fairnustar facility. International Journal of Modern Physics E 2006;15(08):1979-88.

URL: https://doi.org/10. 1142/S0218301306005484. doi $10.1142 / \mathrm{S} 0218301306005484$. arXiv:https://doi.org/10.1142/S0218301306005484

[2] Kalantar-Nayestanaki N, Bruce A. Nustar: Nuclear structure astrophysics and reactions at fair. Nuclear Physics News $\quad 2018 ; 28(3): 5-11$

URL: https://doi.org/10.1080/ 10619127.2018 .1495476 . doi $10.1080 / 10619127.2018 .1495476$ arXiv:https://doi.org/10.1080/10619127.2018.1495476.

[3] Geissel H, Weick H, Winkler M, Mnzenberg G, Chichkine V, Yavor M, et al. The super-frs project at gsi. Nuclear Instruments and Methods in Physics Research Section B: Beam Interactions with Materials and Atoms 2003;204:71 - 85. URL: http://www.sciencedirect. 10 com/science/article/pii/S0168583X02018931. doi/https://doi.org/ 10.1016/S0168-583X (02)01893-1, 14th International Conference on Electromagnetic Isotope Separators and Techniques Related to their Applications.

[4] Li G, Lizarazo C, Gerl J, Kojouharov I, Schaffner H, Grska $\mathrm{M}$, et al. Simulated characteristics of the DEGAS $\gamma$-detector array. Nuclear Instruments and Methods in Physics Research 355 Section A: Accelerators, Spectrometers, Detectors and Associated a Equipment 2018;890:148 -54. URL: http://www.sciencedirect. 1. com/science/article/pii/S016890021830216X. doi/https://doi.org/ $10.1016 /$ j.nima.2018.02.062. 
[5] Tain J, Algora A, Agramunt J, Guadilla V, Jordan M, Montaner-

[7] Lozeva R, Stuchbery A, Gerl J, Georgiev G, Mertzimekis T, the gSPEC 375

[8] Rudigier M, Podolyak Z, Regan P, Bruce A, Lalkovski S, Canavan R, et al. Fatima - fast timing array for despec at fair. Nuclear Instruments

//www.sciencedirect.com/science/article/pii/S0168900220304332 doi/https://doi.org/10.1016/j.nima.2020.163967.

[9] Braga D, Coleman-Smith PJ, Davinson T, Lazarus IH, Page RD, Thomas Piz A, et al. A decay total absorption spectrometer for despec at fair. Nuclear Instruments and Methods in Physics Research Section A: Accelerators, Spectrometers, Detectors and Associated Equipment 2015;803:36 - 46. URL: http://www.sciencedirect. com/science/article/pii/S016890021501058X. doi/https://doi.org/ 10.1016/j.nima.2015.09.009.

[6] Tolosa-Delgado A, Agramunt J, Tain J, Algora A, Domingo-Pardo C, Morales A, et al. Commissioning of the briken detector for the measurement of very exotic -delayed neutron emitters. Nuclear Instruments and Methods in Physics Research Section A: Accelerators, Spectrometers, Detectors and Associated Equipment 2019;925:133 -47. URL: http://www.sciencedirect. com/science/article/pii/S0168900219301743, doi/https://doi.org/ 10.1016/j.nima.2019.02.004. and Methods in Physics Research Section A: Accelerators, Spectrometers, Detectors and Associated Equipment 2020;969:163967. URL: http: S. Aida: A 16-channel amplifier asic to read out the advanced implantation detector array for experiments in nuclear decay spectroscopy. In: 2011 
2nd International Conference on Advancements in Nuclear Instrumentation, Measurement Methods and their Applications. 2011, p. 1-5.

[10] Bruyneel B, Reiter P, Pascovici G. Characterization of large volume hpge detectors. part ii: Experimental results. Nuclear Instruments and Methods in Physics Research Section A: Accelerators, Spectrometers, Detectors and Associated Equipment 2006;569(3):774 -89.

[11] Pfützner M, Armbruster P, Baumann T, Benlliure J, Bernas M, Catford $\mathrm{W}$, et al. New isotopes and isomers produced by the fragmentation of $238 \mathrm{U}$ 395 a at $1000 \mathrm{Mev} /$ nucleon. Physics Letters B 1998;444(1):32 -7. URL: http: //www.sciencedirect.com/science/article/pii/S037026939801332X. doi:https://doi .org/10.1016/S0370-2693(98)01332-X.

[12] Saha S, Palit R, Sethi J, Biswas S, Singh P. Geant4 simulation study of indian national gamma array at tifr. Journal of Instrumentation 2016;11(03):P03030.

[13] Saha S, Arici T, Gerl J, Grska M, Pietralla N, Davinson T, et al. On the $\beta$-detection efficiency of a combined si and plastic stack detector for despec. Nuclear Instruments and Methods in Physics Research Section A: Accelerators, Spectrometers, Detectors and Associated Equipment 2020;975:164196. URL: http://www.sciencedirect. a com/science/article/pii/S0168900220305921. doi/https://doi.org/ 10.1016/j.nima.2020.164196. 